Research Article

\title{
Comparison of Mid-Upper-Arm Circumference and Weight-For-Height $Z$-Score in Identifying Severe Acute Malnutrition among Children Aged 6-59 Months in South Gondar Zone, Ethiopia
}

\author{
Dereje Birhanu Abitew $\mathbb{D}^{1},{ }^{1,2}$ Alemayehu Worku Yalew $\mathbb{D}^{1},{ }^{1}$ Afework Mulugeta Bezabih $\mathbb{D}^{3},^{3}$ \\ and Alessandra N. Bazzano (iD) \\ ${ }^{1}$ School of Public Health, Addis Ababa University, Addis Ababa, Ethiopia \\ ${ }^{2}$ School of Public Health, Bahir Dar University, Bahir Dar, Ethiopia \\ ${ }^{3}$ School of Public Health, Mekelle University, Mekelle, Ethiopia \\ ${ }^{4}$ School of Public Health and Tropical Medicine, Tulane University, New Orleans, LA, USA
}

Correspondence should be addressed to Dereje Birhanu Abitew; derejefrae2014@gmail.com

Received 24 August 2020; Revised 1 February 2021; Accepted 20 April 2021; Published 5 May 2021

Academic Editor: Abdah Md Akim

Copyright $(0) 2021$ Dereje Birhanu Abitew et al. This is an open access article distributed under the Creative Commons Attribution License, which permits unrestricted use, distribution, and reproduction in any medium, provided the original work is properly cited.

\begin{abstract}
Children with severe acute malnutrition (SAM) are identified for admission to outpatient therapeutic programs using mid-upperarm circumference (MUAC) or weight for height (WHZ). However, MUAC and WHZ do not identify the same children, and such observed differences might have programmatic implications of missed nutrition therapy if only MUAC is used to identify children with SAM. The objective of the study was to assess any difference in prevalence and degree of agreement between MUAC and WHZ in identifying SAM affected children. A cross-sectional study was conducted in South Gondar Zone, Ethiopia, among 17 districts, with 3 districts and 10 health centers with their clustered health posts selected randomly. A total of 2,040 children were recruited, and data were collected using a parent questionnaire then entered into EpiData and analyzed using SPSS v 20. A total of 1,980 respondents (97.1\%) were interviewed, all of whom were female and rural residents. Children's mean age in months was 23.2 ( $\mathrm{SD} \pm 9.7)$, and $54 \%$ were male children. The prevalence of SAM based on MUAC $<11.5 \mathrm{~cm}$ was $11.2 \%$ (95\% CI: 9.9-12.7) and $11.0 \%$ (95\% CI: 9.7-12.5) based on WHZ <-3. The agreement between MUAC and WHZ was good $(k=0.729)$. The proportion of children with SAM identified using both MUAC and WHZ was $61.2 \%$. The prevalence of SAM identified using both MUAC and WHZ was comparable. A substantial degree of agreement between MUAC and WHZ was observed to diagnose SAM. Therefore, MUAC can be used as an appropriate tool in identifying children with SAM for admission into the outpatient therapeutic program (OTP) in the study area.
\end{abstract}

\section{Introduction}

Undernutrition is the result of nutrient deprivation either due to lack of adequate intake or repeated infection [1] and is categorized as chronic or acute malnutrition based on the duration of deprivation [2]. Acute malnutrition is further classified as moderate acute malnutrition (MAM) or severe acute malnutrition (SAM) based on the degree of malnutrition and the presence of edema [2]; therefore, it is SAM if weight-for-length/height $z$-score (WHZ) is below $-3 \mathrm{SD}$ and/or with MUAC $<115 \mathrm{~mm}$ and/or with bilateral edema and moderately acutely malnourished (MAM) if WHZ is between -2 and -3 or MUAC between 115 and $125 \mathrm{~mm}$ $[3,4]$.

According to a 2018 report, globally over 49 million children under age five were wasted and nearly 17 million were severely wasted [5], while the magnitude nationally in Ethiopia and Amhara Region was found to be $7.2 \%$ and 
7.6\%, respectively [6]. Children with severe acute malnutrition have a high risk of death exceeding 9-fold compared to well-nourished children $[7,8]$. Worldwide, about one million children die every year from SAM $[9,10]$, and in Ethiopia, about $57 \%$ of all under-five deaths are related to malnutrition, of which three-quarters result from complications associated with mild-to-moderate malnutrition [11].

Acutely malnourished children (with severe or moderate malnutrition) were traditionally identified using WHZ, particularly in primary healthcare settings, but measurement of height and weight are often challenging, especially for inexperienced health professionals and community healthcare workers, as this needs technical and practical skills; therefore, MUAC, a relatively simple procedure using simple colored plastic, was introduced as an alternative $[8,12,13]$, and in the currently updated SAM management protocols, MUAC $<115 \mathrm{~mm}$ or WHZ $<-3 \mathrm{SD}$ are used as independent indicators for the identification of SAM [14].

Even if both WFH and MUAC can identify children with SAM [2], there are arguments about which may identify more SAM cases. Some studies have reported that a relatively similar magnitude of acutely malnourished children are identified if MUAC or WHZ is used [2, 15-20], while other studies reported that more SAM cases are identified if WHZ is used than MUAC $[21,22]$. On the other hand, more SAM cases were reportedly detected if MUAC was used rather than WHZ [23].

Poor level of agreement between MUAC and WHZ in identifying SAM cases has also been reported ranging from 0 to $54 \%[2,15,16,18,22,24-26]$. Moreover, the discrepancy also varies in terms of child age, sex, and residential area [2, 27, 28].

Such observed differences might have programmatic implications in that depending on whether MUAC or WHZ is used in identification, and then acutely malnourished children could unreasonably miss nutrition therapy [2]. Therefore, the objective of this study was to assess differences in prevalence and degree of agreement between WHZ and MUAC in identifying SAM affected children aged 6 to 59 months in South Gondar Zone, Amhara Region, Ethiopia.

\section{Methods and Materials}

The full and complete methods for the study have been previously described in a publication related to "Predictors of relapse of acute malnutrition following exit from community-based management program in Amhara Region, northwest Ethiopia: An unmatched case-control study" [29] and in a previous publication related to risk of SAM following exit from care titled "Rural children remain more at risk of acute malnutrition following exit from communitybased management of acute malnutrition program in South Gondar Zone, Amhara Region, Ethiopia: A comparative cross-sectional study" [30].

2.1. Study Design and Setting. This was a community-linked, facility-based cross-sectional study conducted from 10 November 2017 to 30 January 2018 in the South Gondar Zone of Amhara Region, Ethiopia. The zone has 17 districts, five of which are town administrations. Debretabor is the capital city of the zone situated about $100 \mathrm{Km}$ east of Bahir Dar (the capital city of Amhara Region) and $667 \mathrm{~km}$ north of Addis Ababa (the capital city of Ethiopia). According to the Government of Ethiopia, the 2017/18 population of the zone was $2,484,929$ of which 183,525 were children $0-4$ yrs old [31]. For more detail of the study area and setting, see previous references $[29,30]$.

2.2. Population and Inclusion/Exclusion Criteria. The sampling frame for this study was children aged 6-59 months who had been discharged as recovered from the community-based management of acute malnutrition (CMAM) program and age-matched children who had never been treated for SAM in South Gondar Zone, Amhara Region, Ethiopia. The study population constituted children aged 6-59 months in the randomly selected districts of South Gondar Zone. Children aged 6-59 months in randomly selected districts were included, while children with chronic illnesses or congenital malformations or with visible spinal deformity were excluded. Additional details of how the study population was selected, and the detailed inclusion and exclusion criteria, can be found in previous publications $[29,30]$.

\subsection{Sample Size Determination and the Sampling Procedure.} The sample size for this study was 2,040 based on the calculation for the two objectives of the project: 1,318 sample size taking 95\% CL, $16.9 \%$ relapse of SAM [32], 3\% margin of error, design effect of 2 , and $10 \%$ nonresponse rate, and for the other objective, taking the percent of acute malnutrition among recovered children following exit from CMAM, 78\% [33], with an assumption of a $10 \%$ difference in the children age 6-59 months who were never treated for SAM, with $95 \%$ CL, $80 \%$ power, a ratio of $1: 1$, design effect of 2 , and including $10 \%$ nonresponse rate, the final sample size for the comparison group was 722 , and therefore, the final sample size was 2,040. The outcome variable was severe acute malnutrition (yes vs. no), while the independent variables were demographic, socioeconomic, household hygiene/ sanitation, awareness of recommended caring and feeding practices for children, health facility access, and household food security status. Regarding the sampling procedure, a two-stage sampling technique was used. Among the 17 districts, 3 rural administrative districts (Ebnat, TachGayint, and Lay-Gayint) were selected, within which 10 health centers with their clustered health posts were selected. Finally, through these identification procedures, mothers/ caretakers of children were contacted by HEWs and asked to bring the children for assessment. The details of the sample size calculation and the sampling procedure can also be found in previous publications $[29,30]$.

\section{Operational Definitions}

3.1. Severe Acute Malnutrition (SAM). A child was considered as severely acutely malnourished if he/she had MUAC $<11.5 \mathrm{~cm}$ or WHZ $<-3$ and/or had bilateral edema [2]. 
3.2. Safe Child Feces Disposal. Child feces disposal was considered safe if the child useda latrine or child feces was rinsed into a latrine and considered unsafe otherwise.

3.3. Improved Water Source. A household was considered to have improved drinking water if the source was either from the pipe, protected spring, protected well, and/or boiled water.

3.4. Good Hand Washing Practice. A respondent was categorized as having good hand washing practice if they reported washing hands at 3 or more critical times/points (before eating/feeding a child, before preparing food/ cooking, after defecation, and after cleansing the child's bottom) [34].

3.5. Household Food Insecurity Status. Food security was determined using the 9-item Household Food Insecurity Access (HFIA) scale questions. Before assigning the food insecurity category (access), each frequency of occurrence responses was coded as 0 for all cases where the answer to the corresponding occurrence question was "no," and then, the four food security categories were computed and created sequentially into the HFIA category 1 as food secure, category 2 as mildly food insecure, category 3 as moderately food insecure, and category 4 as severely food insecure according to FANTA recommendation [35]. Finally, HFIA category 1 was considered as food secure and the remaining as food insecure.

3.6. Data Collection Tools and Measurements. The data collection tools used consisted of a checklist and a questionnaire that were prepared using the therapeutic program (SC/OTP) multichart which is utilized throughout the country [36]. Additionally, the questionnaire used for this study was adapted from validated, locally used questionnaires in nutrition research and survey reports such as the Ethiopian Demographic and Health Survey report [37]. Moreover, questions to assess HH's food security status were taken from a validated questionnaire developed by the Food and Nutrition Technical Assistant (FANTA) project [35].

Mothers were interviewed using the questionnaire which took approximately 20-30 minutes. The data collection took place from 10 November 2017 to 30 January 2018. A total of 15 data collectors were recruited and trained for 2 years, with training content mainly focusing on anthropometric measurement techniques $[2,36]$. The data collectors were closely supervised by the 3 trained health professionals and by the principal investigator.

3.7. Anthropometric Measurements. A wooden measuring board was used for measuring length/height which was measured in recumbent and/or standing position based on their age and was recorded to the nearest $0.1 \mathrm{~cm}$. Children's weight was also taken using a salter/beam balance scale based on their age. Child MUAC was measured using a nonstretchable tape according to the standard and recorded to the nearest $0.1 \mathrm{~cm}$. The presence of edema was assessed and recorded according to accepted standards $[2,36]$. The details of the data collection tools, how they were validated, how the measurements were taken, the operational definitions used, and how data quality was assured can be found in $[29,30]$.

3.8. Data Management Analysis Ethical Issues. The questionnaire was checked manually for completeness and was entered into EpiData version 3.3.2 and exported to SPSS version 20 for analysis. Indices were generated according to the WHO 2006 Child Growth Standards [38] using WHO Anthro software 3.2.2. Descriptive statistics such as mean and standard deviation (SD) were computed for continuous and percentages for categorical variables. The agreement between MUAC and WHZ in identifying acutely malnourished children was estimated by computing weighted kappa. The protocol and consent form were approved by the institutional review board (IRB) of the College of Health Sciences of Addis Ababa University, with an IRB protocol number of 068/16/SPH and meeting number of 001/2917. Written permission letters were obtained from the Regional Health Bureau, South Gondar Zone Health department, and at each District Health Offices of Amhara Region, Ethiopia. Informed verbal consent was obtained from all study respondents after the purpose, risk, benefit, confidentiality, and degree of involvement were fully explained to parents/ caregivers in their local language, and children with MAUC $<11.0 \mathrm{~cm}$ or presence of edema were linked to OTP as this was the currently used admission criterion to therapeutic care.

\section{Results}

4.1. Background Characteristics. Overall, there were 2,040 respondents, of which, 1,980 were interviewed $(97.1 \%$ response rate). All respondents were female gender, rural residents, and Amhara ethnicity. The mean age in years of respondents was $29.1(\mathrm{SD} \pm 6.6)$, and the majority (87.0\%) were in the age range of 20-39 years. The mean age of children in months was $23.2 \pm$ (SD 9.7) and about half (49\%) were in the age range of $12-23$ months, and $54 \%$ were male children. The majority ( $87 \%)$ were farmers by occupation, $82 \%$ had no formal education, and $75 \%$ were food-secure households (see Table 1).

4.2. The Prevalence of Acute Malnutrition Based on MUAC and WHZ. The prevalence of SAM was $11.2 \%$ (95\% CI: 9.9-12.7) based on MUAC $<11.5 \mathrm{~cm}$ and $11.0 \%$ (95\% CI: 9.7-12.5) based on $\mathrm{WHZ}<-3$. The proportion of SAM children identified based on MUAC $<11.0 \mathrm{~cm}$ was $6.1 \%(95 \%$ CI: $5.1-7.3)$ and based on either MUAC $<11.5 \mathrm{~cm}$ or $\mathrm{WHZ}<-3$ was $13.8 \%$ (95\% CI: $12.3-15.4)$. SAM was more common among males than female children using both indicators, but no significant difference was observed in the child age. The overall prevalence of acute malnutrition was 
TABLE 1: Background characteristics of respondents and children aged 6-59 months, South Gondar Zone, Amhara Region, Ethiopia, 2017/ $18(n=1980)$.

\begin{tabular}{|c|c|c|}
\hline Variable & Response category & \# (\%) \\
\hline \multirow{5}{*}{ Respondent age in yrs. } & Mean \pm SD & $29.1 \pm 6.6$ \\
\hline & $15-19$ & $77(3.9)$ \\
\hline & $20-29$ & $916(46.3)$ \\
\hline & $30-39$ & $805(40.7)$ \\
\hline & $40+$ & $182(9.2)$ \\
\hline \multirow{6}{*}{ Child age in months } & Mean \pm SD & $23.2 \pm(9.7)$ \\
\hline & 6-11 & $120(6.1)$ \\
\hline & $12-23$ & $968(48.9)$ \\
\hline & $24-35$ & $627(31.7)$ \\
\hline & $36-47$ & $182(89.2)$ \\
\hline & $48-60$ & $83(4.2)$ \\
\hline \multirow{2}{*}{ Child sex } & Male & $1063(53.7)$ \\
\hline & Female & $917(46.3)$ \\
\hline \multirow{2}{*}{ Respondent education status } & No formal & $1616(81.6)$ \\
\hline & Formal & $364(18.4)$ \\
\hline \multirow{2}{*}{ Respondent occupation } & Farming & $1730(87.4)$ \\
\hline & Other than farming & $250(12.6)$ \\
\hline \multirow{2}{*}{ HH family size } & $\geq 5$ & $1088(54.9)$ \\
\hline & $<5$ & $892(45.1)$ \\
\hline \multirow{2}{*}{$\mathrm{HH}$ food insecure } & No & $1481(74.8$ \\
\hline & Yes & $499(25.2)$ \\
\hline \multirow{2}{*}{ Water source improved } & Yes & $1083(54.7)$ \\
\hline & No & $897(45.3)$ \\
\hline \multirow{2}{*}{ Latrine (pit with or without cover) } & Yes & $1714(86.6)$ \\
\hline & No & $266(13.4)$ \\
\hline \multirow{2}{*}{ Hand washing practice } & Poor & $1077(54.4)$ \\
\hline & Good & $903(45.6)$ \\
\hline \multirow{2}{*}{ Child feces disposal (safe) } & Yes & $1127(56.9)$ \\
\hline & No & $853(43.1)$ \\
\hline \multirow{2}{*}{ BF initiation within $1 \mathrm{hr}$ of birth $(n=1977)$} & Yes & $1283(64.9)$ \\
\hline & No & $694(35.1)$ \\
\hline \multirow{2}{*}{ The practice of prelacteal feeding } & Yes & $107(5.4)$ \\
\hline & No & $1873(94.6)$ \\
\hline \multirow{2}{*}{ Vitamin A supplementation in the past 6 months } & Yes & $1393(70.4)$ \\
\hline & No & $587(29.6)$ \\
\hline \multirow{2}{*}{ History of illness in the past 2 wks. } & Yes & $346(17.5)$ \\
\hline & No & $1634(82.5)$ \\
\hline \multirow{2}{*}{ Preparing food separately for children } & Yes & $1150(58.1)$ \\
\hline & No & 830 (41.9) \\
\hline \multirow{2}{*}{ Another family member on SAM treatment } & Yes & $42(2.1)$ \\
\hline & No & $1938(97.9)$ \\
\hline
\end{tabular}

32.0\% (95\% CI: 30.4-34.1) based on MUAC $<12.5 \mathrm{~cm}$ and 27.3\% (95\% CI: 25.3-29.3) based on $\mathrm{WHZ}<-2$, as illustrated in Tables2 and 3, Figure 1.

4.3. Agreement between MUAC and WHZ in Identifying SAM Children. A weighted Kappa (Cohen's) test was calculated using a 2 by 2 table to determine the level of agreement between MUAC $<11.5$ and $\mathrm{WHZ}<-3$ in identifying SAM children. The overall agreement between MUAC and WHZ was good $(K=0.729)$. The proportion of SAM children identified using both MUAC and WHZ was $61.2 \%$. See Table 4 for further illustration.

\section{Discussion}

Currently, WHO guidelines recommend the use of low midupper-arm circumference (MUAC $<115 \mathrm{~mm}$ ), low weightfor-height (WFH $<-3 z$-scores of WHO standards), and/or edema as internationally recognized independent diagnostic criteria for severe acute malnutrition in children age 6-59 months $[2,14]$ and at community-based programs; however, it is recommended to use only MUAC and edema as criteria to admit children with SAM to the OTP [8].

In the current study, the ability of the two indicators (MUAC $<11.5 \mathrm{~cm}$ and $\mathrm{WHZ}<-3$ ) to identify SAM children was compared, and the findings indicated that the proportion 
TABle 2: Some of the epidemiologic variables from the two parent studies [29, 30].

\begin{tabular}{|c|c|c|c|}
\hline Variable & Response & $\begin{array}{c}\text { Following recovery } \\
\quad(n=1273) \\
\text { Frequency }(\%) \\
\end{array}$ & $\begin{array}{c}\text { Comparison group of reference } 30 \\
(n=707) \\
\text { Frequency }(\%)\end{array}$ \\
\hline \multirow{2}{*}{ Child sex } & Male & $680(53.4)$ & $383(54.2)$ \\
\hline & Female & $593(46.6)$ & $324(45.8$ \\
\hline \multirow{5}{*}{ Child age in month } & $6-11$ & $50(3.9)$ & $70(9.9)$ \\
\hline & $12-23$ & $689(54.1)$ & $279(39.5)$ \\
\hline & $24-35$ & $387(30.4)$ & $240(33.9)$ \\
\hline & $36-47$ & $109(8.6)$ & $73(10.3$ \\
\hline & $48-59$ & $38(3.0)$ & $45(6.4)$ \\
\hline \multirow{3}{*}{ Respondent education status } & Unable to read and write & $768(60.3)$ & $404(57.1)$ \\
\hline & Read and write only & $292(22.9)$ & $152(21.5)$ \\
\hline & Primary and above & $213(16.7)$ & $151(21.4)$ \\
\hline \multirow{2}{*}{ Respondent occupation } & Farming & $1188(93.3)$ & $542(76.7)$ \\
\hline & Other than farming & $85(6.7)$ & $165(23.3)$ \\
\hline \multirow{2}{*}{ HH family size } & $<5$ & $558(43.8)$ & $334(47.2)$ \\
\hline & $\geq 5$ & $715(56.2)$ & 37352.8() \\
\hline \multirow{2}{*}{$\mathrm{HH}$ food security status } & Insecure & $321(25.2)$ & $178(25.2)$ \\
\hline & Secure & $952(74.8)$ & $529(74.8)$ \\
\hline \multirow{2}{*}{ Vaccinated for measles } & No & $58(4.6)$ & $105(14.9)$ \\
\hline & Yes & $1215(95.4)$ & $602(85.1)$ \\
\hline \multirow{2}{*}{ Vitamin A supplemented in the past 6 months } & No & $318(25.0)$ & $269(38.0 \%)$ \\
\hline & Yes & $955(75.0)$ & $438(62.0)$ \\
\hline \multirow{2}{*}{ Water source for drinking improved } & No & $598(47.0)$ & $299(42.3)$ \\
\hline & Yes & $675(53.0)$ & $408(57.7)$ \\
\hline \multirow{2}{*}{ Type of latrine } & Pit & $1105(86.8)$ & $609(86.1)$ \\
\hline & Open field/bush & $168(13.2)$ & $98(13.9)$ \\
\hline \multirow{2}{*}{ Hand washing practice } & Poor & $584(45.9)$ & $319(45.1)$ \\
\hline & Good & $689(54.1)$ & $388(54.9)$ \\
\hline \multirow{2}{*}{ Safe child feces disposal practice } & No & $570(44.8)$ & $283(40.0)$ \\
\hline & Yes & $703(55.2)$ & $424(60.0)$ \\
\hline \multirow{2}{*}{ HH own farmland } & No & $77(6.0)$ & $106(15.0)$ \\
\hline & Yes & $1196(94.0)$ & $601(85.0)$ \\
\hline \multirow{2}{*}{ HH own animals } & No & $95(7.5)$ & $117(16.5)$ \\
\hline & Yes & $1178(92.5)$ & $590(83.5)$ \\
\hline
\end{tabular}

of SAM affected children identified by the two indicators (MUAC $<11.5 \mathrm{~cm}$ and $\mathrm{WHZ}<-3$ ) were comparable, while a lower proportion of affected children were identified based on the admission criteria $($ MUAC $<11.0 \mathrm{~cm}$ ) used at the data collection time compared with the recommended criteria (MUAC $<11.5 \mathrm{~cm}$ and $\mathrm{WHZ}<-3$ ) [14]. The finding supports the WHO and UNICEF 2009 report where the prevalence of SAM based on MUAC $<11.5 \mathrm{~cm}$ and WHZ $<-3$ was very similar [2]. Similar findings were reported among Nigerian children with SAM [16]. A systematic review has also reported that MUAC performed at least as well as measures of $\mathrm{W} / \mathrm{H}$ to identify SAM children [39]. A study in Southern Ethiopia indicated a nonsignificant difference in the prevalence of SAM based on MUAC and WHZ [23]. However, a study in Pakistan identified more children with SAM by MUAC compared to WHZ [20]. Moreover, two studies in Niger reported that more cases were identified using MUAC than WHZ $[24,40]$. But, a study in Sudan indicated that more SAM cases were identified using WHZ than with MUAC [21]. A study in South Africa also reported the identification of more children with SAM with WHZ than using MUAC [15].
When considering the prevalence of global acute malnutrition (GAM) $($ MUAC $<12.5 \mathrm{~cm}$ or WHZ $<-2)$, MUAC identified more acutely malnourished children compared with WHZ. The finding was in agreement with a study conducted in Niger that more acutely malnourished children were identified using MUAC than WHZ [40]. Also, a study in Southern Ethiopia described that MUAC categorized more children as wasted compared with WHZ [23]. On the other hand, a study in South Africa reported $\mathrm{W} / \mathrm{H}$ to be more sensitive than MUAC to identify acutely malnourished children [15]. Moreover, a study in Somalia revealed that GAM was higher based on WHZ than based on MUAC [41].

The proportion of acute malnutrition (SAM or MAM) identified based on MUAC and WHZ has also been reported by analyzing anonymous data from 1,832 anthropometric surveys from 47 countries indicating that only a minority of children were diagnosed as malnourished using these criteria. Moreover, the magnitude and direction of discrepancy varied dramatically between countries, with some having most children diagnosed malnourished by MUAC and in others by WHZ alone [25]. 
TABLE 3: Magnitude of acute malnutrition based on MUAC in $\mathrm{cm}$ and WHZ among children, South Gondar Zone, Ethiopia, 2017/18 $(n=1980)$.

\begin{tabular}{|c|c|c|c|c|c|c|c|}
\hline & & \multicolumn{4}{|c|}{ Severe acute malnutrition (SAM) } & \multicolumn{2}{|c|}{$\begin{array}{l}\text { Global acute malnutrition } \\
\text { (GAM) }\end{array}$} \\
\hline & e in month & $\begin{array}{c}\text { MUAC <11.5 } \\
\%(95 \% \mathrm{CI})\end{array}$ & $\begin{array}{c}\mathrm{WHZ}<-3 \\
\%(95 \% \mathrm{CI})\end{array}$ & $\begin{array}{c}\text { MUAC }<11.5 \text { and } \\
\text { WHZ }<-3 \\
\%(95 \% \mathrm{CI})\end{array}$ & $\begin{array}{c}\text { MUAC }<11.5 \text { or } \\
\text { WHZ }<-3 \\
\%(95 \% \mathrm{CI})\end{array}$ & $\begin{array}{c}\text { MUAC < } 12.5 \\
\%(95 \% \mathrm{CI})\end{array}$ & $\begin{array}{c}\text { WHZ<-2 } \\
\%(95 \% \mathrm{CI})\end{array}$ \\
\hline \multirow{3}{*}{$6-23$} & $\begin{array}{c}\text { Combined } \\
(1088)\end{array}$ & $\begin{array}{c}11.5 \\
(9.6-13.4)\end{array}$ & $9.9(8.2-11.9)$ & $7.8(6.3-9.6)$ & $13.5(11.5-15.7)$ & $\begin{array}{c}33.5 \\
(30.7-36.4)\end{array}$ & $26.9(24.3-29.7)$ \\
\hline & Male (559) & $\begin{array}{c}14.1 \\
(11.4-17.3)\end{array}$ & $\begin{array}{c}13.8 \\
(11.0-16.9)\end{array}$ & $11.7(8.3-13.6)$ & $17.2(14.1-20.6)$ & $\begin{array}{c}39.5 \\
(35.5-43.7)\end{array}$ & $32.2(28.3-36.2)$ \\
\hline & Female (529) & $8.5(6.3-11.2)$ & $5.9(4.0-8.2)$ & $4.7(3.1-6.9)$ & $9.6(7.3-12.3)$ & $\begin{array}{c}27.2 \\
(23.5-31.2)\end{array}$ & $21.4(17.9-25.1)$ \\
\hline \multirow{3}{*}{$24-59$} & $\begin{array}{l}\text { Combined } \\
(892)\end{array}$ & $\begin{array}{c}11.0 \\
(9.0-13.2)\end{array}$ & $\begin{array}{c}12.3 \\
(10.2-14.7)\end{array}$ & $9.2(7.4-11.3)$ & $14.1(11.9-16.6)$ & $\begin{array}{c}30.2 \\
(27.2-33.3)\end{array}$ & $27.7(24.8-30.8)$ \\
\hline & Male (504) & $\begin{array}{c}12.3 \\
(9.6-15.5)\end{array}$ & $\begin{array}{c}14.3 \\
(11.3-17.8)\end{array}$ & $10.5(8.0-13.5)$ & $16.1(13.0-19.6)$ & $\begin{array}{c}31.7 \\
(27.7-36.0)\end{array}$ & $29.0(25.0-33.1)$ \\
\hline & Female (388) & $9.3(6.6-12.6)$ & $9.8(7.0-13.2)$ & $7.5(5.1-10.6)$ & $11.6(8.6-15.2)$ & $\begin{array}{c}28.1 \\
(23.7-32.8)\end{array}$ & $26.0(21.7-30.7)$ \\
\hline \multirow{3}{*}{$6-59$} & Male (1063) & $\begin{array}{c}13.3 \\
(11.3-15.5)\end{array}$ & $\begin{array}{c}14.0 \\
(12.0-16.3)\end{array}$ & $10.6(8.8-12.6)$ & $16.7(14.5-19.0)$ & $\begin{array}{c}35.8 \\
(33.0-38.8)\end{array}$ & $30.7(27.9-33.5)$ \\
\hline & Female (917) & $8.8(7.1-10.9)$ & $7.5(5.9-9.4)$ & $5.9(4.5-7.6)$ & $10.5(8.6-12.6)$ & $\begin{array}{c}27.6 \\
(24.7-30.6)\end{array}$ & $23.3(20.6-26.2)$ \\
\hline & Total (1980) & $\begin{array}{c}11.2 \\
(9.9-12.7)\end{array}$ & $\begin{array}{c}11.0 \\
(9.7-12.5)\end{array}$ & $8.4(7.2-9.7)$ & $13.8(12.3-15.4)$ & $\begin{array}{c}32.0 \\
(30.0-34.1)\end{array}$ & $27.3(25.3-29.3)$ \\
\hline
\end{tabular}

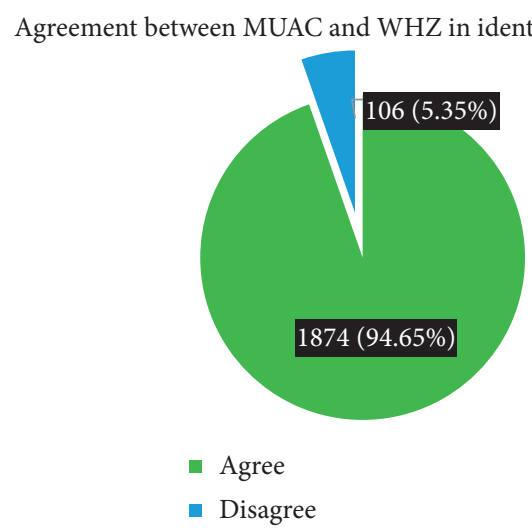

FIGURE 1: A figure showing frequencies and the corresponding proportions for agreement and disagreement between MUAC vs. WHZ in identifying SAM children following recovery from CMAM, South Gondar Zone, Amhara Region, 017/18 $(n=1980)$.

TABle 4: Agreement between MUAC and WFH in Z-score in identifying SAM children by age and sex, South Gondar, Amhara Region, Ethiopia, 2017/18 $(n=1980)$.

\begin{tabular}{|c|c|c|c|}
\hline & SAM $(\mathrm{WHZ}<-3)$ & Not SAM $(\geq-3)$ & Total \\
\hline \multicolumn{4}{|l|}{ All children } \\
\hline SAM (MUAC <11.5) & 167 & 55 & 222 \\
\hline Not SAM $($ MUAC $\geq 11.5$ & 51 & 1707 & 1758 \\
\hline Total & 218 & $\begin{array}{c}1762 \\
K=0.729\end{array}$ & 1980 \\
\hline
\end{tabular}

kap MUAC_Kappa WHZ_Kappa, wgt (w)

Ratings weighted by:

$1.0000 \quad 0.0000$

$0.0000 \quad 1.0000$

\begin{tabular}{|c|c|c|c|c|c|}
\hline Agreement (\%) & Expected agreement (\%) & Kappa & Std. Err. & $Z$ & Prob $>Z$ \\
\hline 94.65 & 80.25 & 0.7290 & 0.0225 & 32.44 & 0.0000 \\
\hline
\end{tabular}


The variation in the prevalence of acute malnutrition (both SAM and MAM) based on these two indicators (MUAC and WHZ) could, therefore, be associated with different aspects such as body composition. This may be because WHZ is more influenced by body shape than MUAC. For example, a study in Ethiopia considering children from agrarian and pastoralist areas identified that agrarian children were having higher sitting to standing ratio of height (SSR) values than pastoralist children. Therefore, WHZ may not be more affected by SSR in agrarian than in pastoralist (long-legged) children. Thus, WHZ and MUAC yielded similar estimates in agrarian but different estimates in pastoralist children [17]. A nonsignificant but high proportion of SAM children both using MUAC and WHZ and a high proportion of GAM based on MUAC compared with WHZ, therefore, may be associated with the agrarian nature of the study area. The variation in the prevalence of acute malnutrition may also be due to children's stunting status; a study has shown that stunted children tend to accumulate more fat mass and gain less lean body mass than nonstunted children and, therefore, perhaps, leading to lower MUAC cutoffs [42]. The higher stunting prevalence (41.3\%) in the Amhara Region [6] and in the current study area (69\%) could, therefore, have an effect on the prevalence estimate of acute malnutrition using these two indicators.

To examine the strength of agreement between MUAC and WHZ in identifying SAM children, Cohen's kappa (k) was used. The agreement between MUAC $<11.0 \mathrm{~cm}$ and $\mathrm{WHZ}<-3$ in identifying SAM children was marginally poor $(K=0.600)$, and the proportion of SAM children identified by both indicators was below half $(46.1 \%)$, whereas the degree of agreement between the WHO-recommended MUAC cutoffs (MUAC $<11.5 \mathrm{~cm}$ ) and $\mathrm{WHZ}<-3$ was substantial $(K=0.729)$ and a higher proportion of SAM children were identified by both indicators (61.2\%). This may indicate that admission cutoff used at the data collection time (MUAC $<11.0 \mathrm{~cm}$ ) has been missed in identifying more SAM children than the WHO recommendation to change from MUAC $<11.0 \mathrm{~cm}$ to MUAC $<11.5 \mathrm{~cm}$ to increase diagnostic accuracy [43].

The agreement based on MUAC $<11.5 \mathrm{~cm}$ was comparable to a study conducted in Southern Ethiopia with $71 \%$ agreement between MUAC $<115 \mathrm{~mm}$ and WHZ $<-3$ [44]. Other studies also reported agreement in the two indicators. A study in the rural Gambia reported a $59.8 \%$ overlap between WHZ and MUAC in identifying SAM children [45]. The WHO and UNICEF report in 2009 indicated a $40 \%$ agreement in identifying SAM children using WHZ and MUAC [2], and in Niger, 39\% agreement in SAM identification was reported [24]. On the other hand, a poor level of agreement between MUAC and WHZ was reported in Cambodia that SAM screening using $M U A C<115 \mathrm{~mm}$ would have missed over $90 \%$ of children with $\mathrm{WHZ}<-3$; conversely, WHZ $<-3$ missed $80 \%$ of children with MUAC $<115 \mathrm{~mm}$ [27]. Furthermore, a study in Nigeria also reported that none of the children classified as SAM by WHZ were classified as SAM by MUAC [16]. Despite discrepancies among study findings, the admission criteria at data collection time could miss more SAM children, while the current to-be-used community-based screening using MUAC $<11.5 \mathrm{~cm}$ to admit to OTP program is less likely to miss SAM children if only MUAC $<11.5 \mathrm{~cm}$ is used as screening/admission criteria in the study area.

The WHO and UNICEF report in 2009 indicated that children with WHZ below -3 SD based on WHO standards have a high risk of death exceeding 9-fold compared with children with WHZ >-3 [7]. Similarly, children with MUAC below $115 \mathrm{~mm}$ showed an increased risk of dying [8] with a specificity of more than $99 \%$ in children aged 6-59 months in the two indicators [2]. A study in India indicated that MUAC predicted death better (sensitivity: $95.5 \%$, specificity: 25.0\%) than WHZ (sensitivity: $86.4 \%$, specificity: $21.4 \%$ ) [46]. In the current study, children were admitted to OTP based on MUAC $<11.0 \mathrm{~cm}$ and/or edema, and sensitivity and specificity of MUAC $<11.0 \mathrm{~cm}$ against $\mathrm{WHZ}<-3$ in identifying children with SAM was $49 \%$ and $99 \%$, respectively, but sensitivity is $77 \%$ and specificity is $97 \%$ if MUAC $<11.5 \mathrm{~cm}$ was used as admission criteria. This may indicate that the risk of mortality associated with SAM would have been reduced if MUAC $<11.5 \mathrm{~cm}$ was used as a screening and admission criteria than MUAC $<11.0 \mathrm{~cm}$.

Scholars even recommend MUAC cutoffs to be more than $11.5 \mathrm{~cm}$ to identify more $S A M$ cases and, therefore, reduce mortality. A study in India indicated that the sensitivity and specificity of MUAC $<11.5 \mathrm{~cm}$ against $\mathrm{WHZ}<-3$ was $13.6 \%$ and $99.3 \%$, respectively, recommending to increase the cutoffs to $<12.8$, so $50 \%$ sensitivity and $90.8 \%$ specificity [47]. Another study in the Wardha district of India also reported $23.5 \%$ sensitivity and $99.7 \%$ specificity for MUAC $<11.5 \mathrm{~cm}$ and recommending cutoff to be MUAC $<12.8 \mathrm{~cm}$ to diagnose SAM at $74.1 \%$ sensitivity and 93.2\% specificity [48].

Regarding the sex of children, the prevalence of SAM in boys was higher compared with girls both using MUAC and WHZ. However, a study in South Sudan, the Philippines, Chad, and Bangladesh reported that male children were diagnosed more acutely malnourished either by WHZ $<-2$ or MUAC $<125$ but female children were detected as more malnourished by MUAC $<125$ only [49]. But a study in India indicated that MUAC $<115 \mathrm{~mm}$ preferentially selected more girls than boys [50]. A study in southern Ethiopia also showed the prevalence of SAM in boys to be higher than girls when WHZ was used but identified no sex difference when MUAC was used [23].

When considering global acute malnutrition based on MUAC $<12.5 \mathrm{~cm}$ and $\mathrm{WHZ}<-2$, the prevalence was still higher in boys than girls, however this was not consistent with other research findings. For example, a study in Cambodia reported that WHZ identifies more acutely malnourished boys than girls while more females than male children using MUAC [28]. A study in Somalia also revealed that boys to be diagnosed as acutely malnourished by WHZ more than female children while girls were more acutely malnourished than boys using MUAC [41]. Therefore, it may not always be possible to state that MUAC is better at identifying acutely malnourished children more than WHZ, and vice versa.

The high prevalence of acute malnutrition (both SAM and MAM) among male compared with female children 
could be due to the coexistence of stunting. It was reported that acute malnutrition is linked to an increased risk for stunting, so this could limit optimal linear growth due to the occurrence of both wasting and stunting over several months in an individual child [51]. A study in rural Senegal to describe the patterns of concurrent wasting and stunting (WaSt) among children aged 6-59 months and found that WaSt was more highly prevalent (more stunting if wasted, more wasting if stunted) in boys than girls [52]. Descriptive epidemiology of multiple anthropometric deficits using data from 51 countries also indicated that WaSt is more common among male than female children [53], and also, in the current study, male children were found more wasted and stunted than female children $(34.3 \%$ vs. $25.8 \%)$ and were more severely wasted and stunted than female children (15.3\% vs. 10.2\%). In the Ethiopian DHS 2019 report, male children were also found more stunted and wasted than female children [6], indicating that male children are more affected than female children in terms of these two indicators in the study area.

Despite the standardization of anthropometric instruments, intensive training, and close supervision, misclassification of children's nutritional status due to measurement error is potentially a limitation. There may also be age misclassification, as children's age relied on the respondent's recall. However, the use of multistage sampling to select study districts and allocating the sample size to the health posts proportionately, and the use of a clear definition of the outcome of interest and adequate sample size determined following a statistical method could allow findings of the current study to be generalized to the target population (children aged 6-59 months).

In conclusion, the proportion of children with SAM identified using both MUAC $<11.5$ and $\mathrm{WHZ}<-3$ was comparable and a substantial degree of agreement was observed between MUAC and WHZ to diagnose severe acute malnutrition. MUAC has also shown to be more sensitive and specific in identifying SAM affected children. This may have important implications for CMAM programming in that use of only MUAC can still be an appropriate tool in identifying SAM in children for admission into the outpatient therapeutic program in the study area.

\section{Abbreviations}

CMAM: Community-based management of acute malnutrition

DHS: Demographic health survey

FANTA: Food and nutrition technical assistance

GAM: Global acute malnutrition

HEW: Health extension worker

HFA: Height for age

HFIAS: Household food insecurity access score

HP: $\quad$ Health post

IRB: Institutional review board

MAM: Moderate acute malnutrition

MUAC: Mid-upper-arm circumference

OTP: $\quad$ Outpatient therapeutic program

SAM: $\quad$ Severe acute malnutrition
SC: $\quad$ Stabilization centre

SSR: $\quad$ Sitting to standing ratio

UNICEF: United Nations Children's Fund

WaSt: Wasting and stunting

WFA: Weight for age

WFH: Weight for height

WHO: World Health Organization.

\section{Data Availability}

The data used to support the findings of this study are available from the corresponding author upon request.

\section{Conflicts of Interest}

The authors declare that they have no conflicts of interest.

\section{Acknowledgments}

This study was funded by Addis Ababa University, School of Public Health.. But, the funder had no role in study design, data collection, and analysis, decision to publish, or preparation of the manuscript. The authors' appreciation goes to the Amhara Regional Health Bureau, South Gondar Zone Health Department, and all the three district health office heads and nutrition focal persons. The authors also specially thank study participants, data collectors, and supervisors.

\section{References}

[1] WHO, Management of Severe Malnutrition: A Manual for Physicians and Other Senior Health Workers, WHO, Geneva, Switzerland, 1999.

[2] WHO and UNICEF, WHO Child Growth Standards and the Identification of SAM in Infants and Children, A Joint Statement by the World Health Organization and the United Nations Children's Fund, World Health Organization Press, Geneva, Switzerland, 2009.

[3] S. Collins and R. Yates, "The need to update the classification of acute malnutrition," The Lancet, vol. 362, no. 9379, 2003.

[4] Sphere-Handbook, Sphere Handbook: Humanitarian Charter and Minimum Standards in Humanitarian Response, Practical Action, 2018.

[5] UNICEF, WHO, and World Bank, "Levels and trends in child malnutrition: key findings of the 2018 edition of the joint child malnutrition estimates," UNICEF, WHO, and World Bank, New York, NY, USA, 2018.

[6] EDHS, Ethiopian Public Health Institute [Ethiopia] and ICF, Ethiopia Mini Demographic and Health Survey: Key Indicators, EPHI and ICF, Rockville, MD, USA, 2019.

[7] R. E. Black, L. H. Allen, Z. A. Bhutta et al., "Maternal and child undernutrition: global and regional exposures and health consequences," The Lancet, vol. 371, no. 9608, pp. 243-260, 2008.

[8] M. Myatt, T. Khara, and S. Collins, "A review of methods to detect cases of severely malnourished children in the community for their admission into community-based therapeutic care programs," Food and Nutrition Bulletin, vol. 27, no. 3, pp. S7-S23, 2006.

[9] UNICEF, UNICEF, Facts for Life, 4th edition, 2010. 
[10] WHO, Essential Nutrition Actions: Improving Maternal, Newborn, Infant and Young Child Health and Nutrition, WHO, Geneva, Switzerland, 2013.

[11] B. Todd, An Assessment of the Causes of Malnutrition in Ethiopia, A Contribution to the Formulation of a National Nutrition Strategy for Ethiopia, 2005.

[12] WHO, Community-based Management of Severe Acute Malnutrition; A Joint Statement by the World Health Organization, the World Food Programme, the United Nations System Standing Committee on Nutrition and the United Nations Children's Fund, World Health Organization, Geneva, Switzerland, 2007.

[13] WHO, UNICEF, and SCN, Informal Consultation on Community-Based Management of Severe Malnutrition in Children, SCN Nutrition Policy, 2006.

[14] WHO, Updates on the Management of Severe Acute Malnutrition in Infants and Children, World Health Organization, Geneva, Switzerland, 2013.

[15] N. Dukhi, B. Sartorius, and M. Taylor, "Mid-upper arm circumference (MUAC) performance versus weight for height in South African children (0-59 months) with acute malnutrition," South African Journal of Clinical Nutrition, vol. 30, no. 2, 2017.

[16] C. John, "Does mid upper arm circumference identify all acute malnourished 6-59 month old children, in field and clinical settings in Nigeria?" South African Journal of Clinical Nutrition, vol. 30, no. 3, 2017.

[17] M. Myatt, A. Duffield, A. Seal, and F. Pasteur, "The effect of body shape on weight-for-height and mid-upper arm circumference based case definitions of acute malnutrition in Ethiopian children," Annals of Human Biology, vol. 36, no. 1, pp. 5-20, 2009.

[18] C. S. Grijalva-Eternod, J. C. Wells, T. Girma et al., "Midupper arm circumference and weight-for-length $z$ scores have different associations with body composition: evidence from a cohort of Ethiopian infants," The American Journal of Clinical Nutrition, vol. 102, no. 3, pp. 593-599, 2015.

[19] V. M. Aguayo, S. Aneja, N. Badgaiyan, and K. Singh, "Mid upper-arm circumference is an effective tool to identify infants and young children with severe acute malnutrition in India," Public Health Nutrition, vol. 18, no. 17, pp. 3244-3248, 2015.

[20] A. Bari, "Comparison of weight-for-height $Z$-score and mid-upper arm circumference to diagnose moderate and severe acute malnutrition in children aged 6-59 months," Pakistan Journal of Medical Sciences, vol. 35, no. 2, p. 337, 2019.

[21] E. Grellety, L. K. Krause, M. Shams Eldin, K. Porten, and $\mathrm{S}$. Isanaka, "Comparison of weight-for-height and mid-upper arm circumference (MUAC) in a therapeutic feeding programme in South Sudan: is MUAC alone a sufficient criterion for admission of children at high risk of mortality?" Public Health Nutrition, vol. 18, no. 14, pp. 2575-2581, 2015.

[22] P. Kumar, "Comparison between weight-for-height $Z$-score and mid upper arm circumference to diagnose children with acute malnutrition in five Districts in India," Indian Journal of Community Medicine: Official Publication of Indian Association of Preventive \& Social Medicine, vol. 43, no. 3, p. 190, 2018.

[23] A. Tadesse, E. Tadesse, Y. Berhane, and E.-C. Ekström, "Comparison of mid-upper arm circumference and weightfor-height to diagnose severe acute malnutrition: a study in Southern Ethiopia," Nutrients, vol. 9, no. 3, p. 267, 2017.
[24] S. Isanaka, "Comparison of clinical characteristics and treatment outcomes of children selected for treatment of severe acute malnutrition using mid upper arm circumference and/or weight-for-height $z$-score," PLoS One, vol. 10, no. 9, Article ID e0137606, 2015.

[25] E. Grellety and M. H. Golden, "WFH and MUAC should be used independently to diagnose acute malnutrition: policy implications," BMC Nutrition, vol. 2, no. 1, p. 10, 2016.

[26] J. Berkley, I. Mwangi, K. Griffiths et al., "Assessment of severe malnutrition among hospitalized children in rural Kenya," JAMA, vol. 294, no. 5, pp. 591-597, 2005.

[27] A. Laillou, "Optimal screening of children with acute malnutrition requires a change in current WHO guidelines as MUAC and WHZ identify different patient groups," PLoS One, vol. 9, no. 7, Article ID e101159, 2014.

[28] F. Wieringa, L. Gauthier, V. Greffeuille et al., "Identification of acute malnutrition in children in Cambodia requires both mid upper arm circumference and weight-for-height to offset gender bias of each indicator," Nutrients, vol. 10, no. 6, p. 786, 2018.

[29] D. B. Abitew, "Predictors of relapse of acute malnutrition following exit from community-based management program in Amhara region, Northwest Ethiopia: an unmatched casecontrol study," PLoS One, vol. 15, no. 4, Article ID e0231524, 2020.

[30] D. B. Abitew, "Rural children remain more at risk of acute malnutrition following exit from community based management of acute malnutrition program in South Gondar Zone, Amhara Region, Ethiopia: a comparative cross-sectional study," PeerJ, vol. 8, p. e8419, 2020.

[31] CSA, Federal Democratic Republic of Ethiopia, Central Statistical Agency, Population Projection of Ethiopia for All Regions at Wereda Level from 2014-2017, 2013.

[32] S. Burza, R. Mahajan, E. Marino et al., "Seasonal effect and long-term nutritional status following exit from a community-based management of severe acute malnutrition program in Bihar, India," European Journal of Clinical Nutrition, vol. 70, no. 4, pp. 437-444, 2016.

[33] C. Banerjee, M. Hoq, and E. Matin, Follow-up Status of Children with SAM Treated with RUTF in Peri-Urban and Rural Northern Bangladesh, 2016.

[34] WHO, UNICEF, and USAID, Improving Nutrition Outcomes with Better Water, Sanitation and Hygiene: Practical Solutions for Policies and Programmes, New York, NY, USA, 2015.

[35] J. Coates, A. Swindale, and P. Bilinsky, Food and Nutrition Technical Assistance Project (FANTA): Household Food Insecurity Access Scale for Measurement of Food Access: Indicator Guide (V. 3), Food and Nutrition Technical Assistance Project, Washington, DC, USA, 2007.

[36] FMOH, Protocol for the Management of Severe Acute Malnutrition, Federal Ministry of Health, Ethiopia, Addis Ababa, Ethiopia, 2007.

[37] EDHS, Ethiopia Demographic and Health Survey, Addis Ababa, Ethiopia, Demographic and Health Survey, ICF Macro Calverton, Maryland, USA, 2011.

[38] WHO, Child Growth Standard, Department of Nutrition for Health and Development, Geneva, Switzerland, 2007.

[39] L. Platt, P. Easterbrook, E. Gower et al., "Prevalence and burden of HCV co-infection in people living with HIV: a global systematic review and meta-analysis," The Lancet Infectious Diseases, vol. 16, no. 7, pp. 797-808, 2016.

[40] E. Grellety and M. H. Golden, "Severely malnourished children with a low weight-for-height have similar mortality to those with a low mid-upper-arm-circumference: II. 
Systematic literature review and meta-analysis," Nutrition Journal, vol. 17, no. 1, p. 80, 2018.

[41] E. Custodio, "MUAC-for-age more useful than absolute MUAC for nutritional surveillance in Somalia: results from nineteen cross-sectional surveys (2007-2016)," BMC Nutrition, vol. 4, no. 1, p. 8, 2018.

[42] P. A. Martins, D. J. Hoffman, M. T. B. Fernandes et al., "Stunted children gain less lean body mass and more fat mass than their non-stunted counterparts: a prospective study," British Journal of Nutrition, vol. 92, no. 5, pp. 819-825, 2004.

[43] M. A. L. Fernández, P. Delchevalerie, and M. Van Herp, "Accuracy of MUAC in the detection of severe wasting with the new WHO growth standards," Pediatrics, vol. 126, no. 1, pp. e195-e201, 2010.

[44] E. Forsén, E. Tadesse, Y. Berhane, and E.-C. Ekström, "Predicted implications of using percentage weight gain as single discharge criterion in management of acute malnutrition in rural southern Ethiopia," Maternal \& Child Nutrition, vol. 11, no. 4, pp. 962-972, 2015.

[45] A. Burrell, M. Kerac, and H. Nabwera, "Monitoring and discharging children being treated for severe acute malnutrition using mid-upper arm circumference: secondary data analysis from rural Gambia," International Health, vol. 9, no. 4, pp. 226-233, 2017.

[46] A. Chiabi, C. Mbanga, E. Mah et al., "Weight-for-height $Z$ score and mid-upper arm circumference as predictors of mortality in children with severe acute malnutrition," Journal of Tropical Pediatrics, vol. 63, no. 4, pp. 260-266, 2017.

[47] M. Talapalliwar and B. Garg, "Diagnostic accuracy of midupper arm circumference (MUAC) for detection of severe and moderate acute malnutrition among tribal children in Central India," International Journal of Medical Science and Public Health, vol. 5, no. 7, pp. 1317-1321, 2016.

[48] R. Sougaijam, S. Gupta, A. Raut, M. Bharambe, and B. Garg, "Validating the MUAC (Mid-upper arm circumference) cutoff for detection of severe acute malnutrition in children aged 6-59 Months in rural Maharashtra," Indian Pediatrics, vol. 56, no. 3, pp. 209-212, 2019.

[49] D. Roberfroid, "Inconsistent diagnosis of acute malnutrition by weight-for-height and mid-upper arm circumference: contributors in 16 cross-sectional surveys from South Sudan, the Philippines, Chad, and Bangladesh," Nutrition Journal, vol. 14, no. 1, p. 86, 2015.

[50] U. Kapil, R. Pandey, R. Bansal et al., "Mid-upper arm circumference in detection of weight-for-height $Z$-score below -3 in children aged 6-59 months," Public Health Nutrition, vol. 21, no. 10, pp. 1794-1799, 2018.

[51] UNICEF, "Prevention and treatment of severe acute malnutrition in East Asia and the pacific," Report of a Regional Consultation Bangkok, Bangkok, Thailand, 2015.

[52] M. Garenne, "Concurrent wasting and stunting among underfive children in Niakhar, Senegal," Maternal \& Child Nutrition, vol. 15, no. 2, Article ID e12736, 2019.

[53] M. Myatt, "Children who are both wasted and stunted are also underweight and have a high risk of death: a descriptive epidemiology of multiple anthropometric deficits using data from 51 countries," Archives of Public Health, vol. 76, no. 1, p. 28, 2018. 